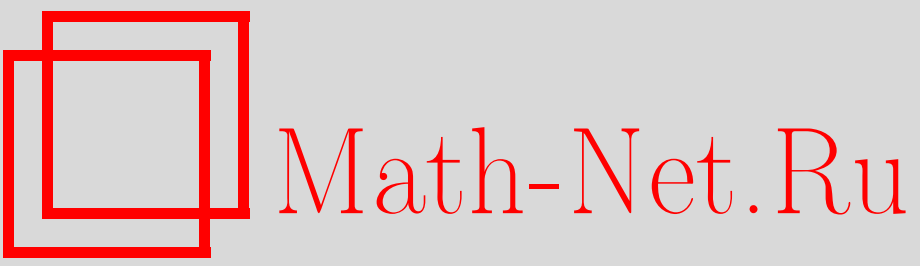

Нгуен Кхак Вьет, О классификации эллиптических расслоений с малым числом особых слоев над базой рода 1, УМН, 1997, том 52, выпуск 6, 175-176

DOI: https://doi.org/10.4213/rm909

Использование Общероссийского математического портала Math-Net.Ru подразумевает, что вы прочитали и согласны с пользовательским соглашением

http://www.mathnet.ru/rus/agreement

Параметры загрузки:

IP : 54.198 .55 .26

26 апреля 2023 г., 17:16:11 


\title{
О КЛАССИФИКАЦИИ ЭЛЛИПТИЧЕСКИХ РАССЛОЕНИЙ С МАЛЫМ ЧИСЛОМ ОСОБЫХ СЛОЕВ НАД БАЗОЙ РОДА 1
}

\author{
K. В. НГуЕН
}

Пусть $k$-некоторое алгебраически замкнутоеполе характеристики $p \neq 2,3$. Пусть $f: X \rightarrow C$ - нетривиальное относительно минимальное якобиево эллиптическое расслоение над $k$ с базой неособой проективной кривой $C$. Пусть $s$ обозначает число особых слоев расслоения $f$. Хорошо известно, что в общем случае $s \geqslant 1$, а в случае $C \simeq \mathbb{P}^{1}$ мы имеем $s \geqslant 2$; если при этом $f$ неизотривиально, то $s \geqslant 3$. В [3] мы классифицировали эллиптические расслоения над $\mathbb{P}^{1} \mathrm{c} s \leqslant 3$. Там же было установлено, что в случае $C \simeq E$ - некоторой эллиптической кривой и $s=1$ расслоение $f: X \rightarrow E$ имеет единственную конфигурацию $\left(I_{6}^{*}\right)$ или ее обратный образ при действии абсолютного морфизма Фробениуса. Наша следующая цель - завершить классификацию в этом случае. Результат этой заметки следует рассматривать как алгебраизацию подобной классификации над комплексными числами, полученную в [5].

Всюду мы фиксируем эллиптическое расслоение $f: X \rightarrow E$ над эллиптической базой $E$, имеющее ровно один особый слой. Типичным примером для этой ситуации служит следующее семейство:

$$
y^{2}=x^{3}-\frac{27 v}{4} x-\frac{27 u}{4}
$$

над базой $E_{0}: u^{2}=v^{3}-1$. Другое семейство получается из (1) “скручиванием" на $\sqrt{v-w}$, где $w^{3}=1$ :

$$
y^{2}=x^{3}-\frac{27 v}{4(v-w)^{2}} x-\frac{27 u}{4(v-w)^{3}} .
$$

Заметим, что кривая $E_{0}$ имеет группу автоморфизмов $G$ порядка 6 и $J$-инвариант указанных вьше семейств индуцирует изоморфизм $E_{0} / G \simeq \mathbb{P}^{1}$. На самом деле, (1) соответствует эллиптической модулярной поверхности $X_{\Gamma}$ над кривой $Y_{\Gamma} \simeq E_{0}$, определенной коммутантом $Г$ группы $S L(2, \mathbb{Z})$, для которой $-1_{2} \notin \Gamma \supset \Gamma(6)(\mathrm{cp} .[4, \S 5],[5])$. Подгруппа $\Gamma$ свободно порождается двумя следующими образующими: $\gamma_{1}=\left(\begin{array}{ll}2 & 1 \\ 1 & 1\end{array}\right), \gamma_{2}=\left(\begin{array}{ll}1 & 1 \\ 1 & 2\end{array}\right)$. Семейство типа (2) оказывается одной из трех (изоморфных) эллиптических модулярных поверхностей, определенных следующими (попарно сопряженными в $S L(2, \mathbb{Z})$ ) подгруппами соответственно: $\left\langle-\gamma_{1}, \gamma_{2}\right\rangle ;\left\langle\gamma_{1},-\gamma_{2}\right\rangle$; $\left\langle-\gamma_{1},-\gamma_{2}\right\rangle$. Эти подгруппы также не содержат $-1_{2}$ и их образы в $P S L(2, \mathbb{Z})$ те же, что для $\Gamma$, а именно, коммутант группы $P S L(2, \mathbb{Z})$. Другими словами, соответствующие модулярные кривые изоморффны $E_{0}$. Заметим, что $J$-инвариант может отождествляться с рациональной функцией $v^{3}$.

Теорема 1. С точностью до изоморфизма семейства (1), (2) исчерпывают все якобиевы әллиптические расслоения $f: X \rightarrow E$, имеющие только один особый слой и ненулевой класс Кодаиры-Спенсера.

Лемма 2. Если якобиево әллиптическое расслоение $f: X \rightarrow E$ имеет ровно один особый слой и ненулевой класс Кодаирь-Спенсера, то этот слой имеет тип I* $J$-инвариант определяет сепарабельный морфизм $J: E \rightarrow \mathbb{P}^{1}$ степени 6.

Тот факт, что особый слой имеет тип $I_{6}^{*}$, следует из гипотезы Шпиро над функциональными полями (cp. [2, Theorem 3] и [3]). Для доказательства второго утверждения рассмотрим разложение $J: E \rightarrow E^{\prime} \stackrel{J^{\prime}}{\longrightarrow} \mathbb{P}^{1}$ на чисто несепарабельную и сепарабелњную части. Затем, применение формулш Римана-Гурвица к $J^{\prime}$ показывает, что $J=J^{\prime}$. 
ЛЕмма 3. Все эллиптические расслоения из леммы 2 имеют одну и ту же базу, которая может быть отождествлена с кривой $E_{0}: u^{2}=v^{3}-1$.

Из леммы 2 легко видеть, что мы имеем следующее описание ветвления: одна точка, полностью разветвленная над $\infty$; над 0 две точки ветвления, каждая с индексом ветвления 3 ; а над 1 три точки ветвления, каждая из которых имеет индекс ветвления 2. Далее, положим $\pi_{1}^{t}:=$ $\pi_{1}^{t}\left(\mathbb{P}^{1}-\{0,1, \infty\}\right)$. Посколшку $\left(p,\left|S_{6}\right|\right)=1$, то, согласно известному результату Гротендика о ручных фундаментальных группах ([SGA, v. 1, XIII, 2.12]) мы видим, что кривая $E$ полностью определена представлением $\pi_{1}^{t}$ в группу перестановок $S_{6}$, образ которого должен быть транзитивной подгруппой в $S_{6}$. Заметим, что это представление по существу описывает, как листы накрытия $J: E \rightarrow \mathbb{P}^{1}$ переставляются. Из описания ветвления выше мы видим, что обход вокруг $\infty$ дает полный цикл; вокруг 0 - произведение трех непересекающихся 2-циклов; а вокруг 1 - произведение двух непересекающихся 3-циклов. Простая комбинаторная проверка показывает, что имеется только одна возможность с точностью до сопряжения (cp. [5, Proposition 3]): $(12)(34)(56) \circ(135)(246)=(145236)$. Имея ввиду семейство (1) (или (2)), мш заключаем, что база $E$ может быть отождествлена с кривой $E_{0}$.

Рассматривая вейерштрассову нормальную форму для расслоения $f: X \rightarrow E_{0}$ из теоремы 1 , видим, что эта нормальная форма также задает эллиптическую поверхность в нулевой характеристике, которая имеет $q=1$ и ровно один особый слой (типа $\left.I_{6}^{*}\right)$. Утверждение теоремы 1 теперь следует из упомянутой классификации (над комплексньпи числами) в [5].

ТЕОРема 4. Пусть $f: X \rightarrow E$ - нетривиальное якобиево семейство эллиптических кривых в характеристике $\neq 2,3$ над некоторой әллиптической базой. Предположим, что $s=1$. Тогда база $E$ может бить отождествлена с кривой $E_{0} u f: X \rightarrow E$ получается из семейств (1) или (2) посредством замены базы с помощью некоторой степени морфизма Фробениуса.

Доказательство сводится к случаю с $K-S(f) \neq 0$ в теореме 1 стандартными рассуждениями $([3], \mathrm{cp} \cdot[1])$

ЗАмечАНИЕ. Для полустабильного эллиптического расслоения $f: X \rightarrow E$, имеющего минимальное число особых слоев $s=2$ и ненулевой класс Кодаиры-Спенсера, известно, что $f$ имеет конфигурацию особых слоев $\left(I_{m}, I_{n}\right)$ с $m+n=12$ (см. [1]). Этот метод доказательства также применяется к этому случаю при условии $p \nmid 6 m n$.

Автор выражает благодарность В. А. Исковских, А. Н. Паршину и Т. Шиоде за поддержку и внимание к работе.

\section{СПИСОК ЛИТЕРАТУРЫ}

[1] Нгуен К. В. Полустабильные эллиптические расслоения с малым числом слоев над базой рода 0 и 1 (в печати). [2] Goldfeld D., Szpiro L. / / Compositio Math. 1995. V. 97. P. 71-87. [3] Nguyen K. V. // Proc. Japan Acad. Ser. A. 1997. V. 73. № 6. P. 103-104. [4] Shioda T. // J. Math. Soc. Japan. 1972. V. 24. P. 20-59. [5] Stiller P. F. // Manuscripta Math. 1988. V. 60. P. 299-321.

Институт Математики Вьетнама

Принято редколлегией 03.09.1997 